\title{
The role of c-FLIP splice variants in urothelial tumours
}

\author{
F Ewald ${ }^{1}$, N Ueffing ${ }^{2,4}$, L Brockmann ${ }^{2}$, C Hader ${ }^{3}$, T Telieps ${ }^{1}$, M Schuster ${ }^{1}$, WA Schulz ${ }^{3}$ and I Schmitz ${ }^{\star, 1}$
}

Deregulation of apoptosis is common in cancer and is often caused by overexpression of anti-apoptotic proteins in tumour cells. One important regulator of apoptosis is the cellular FLICE-inhibitory protein (c-FLIP), which is overexpressed, for example, in melanoma and Hodgkin's lymphoma cells. Here, we addressed the question whether deregulated c-FLIP expression in urothelial carcinoma impinges on the ability of death ligands to induce apoptosis. In particular, we investigated the role of the c-FLIP splice variants $c-F L I P_{\text {long }}\left(c-F L I P_{L}\right)$ and $c-F L I P_{\text {short }}\left(c-F L I P_{S}\right)$, which can have opposing functions. We observed diminished expression of the $c-$ FLIP $_{\mathrm{L}}$ isoform in urothelial carcinoma tissues as well as in established carcinoma cell lines compared with normal urothelial tissues and cells, whereas C-FLIPs was unchanged. Overexpression and RNA interference studies in urothelial cell lines nevertheless demonstrated that $\mathrm{C}$-FLIP remained a crucial factor conferring resistance towards induction of apoptosis by death ligands CD95L and TRAIL. Isoform-specific RNA interference showed C-FLIP $P_{L}$ to be of particular importance. Thus, urothelial carcinoma cells appear to fine-tune C-FLIP expression to a level sufficient for protection against activation of apoptosis by the extrinsic pathway. Therefore, targeting c-FLIP, and especially the c-FLIPL isoform, may facilitate apoptosis-based therapies of bladder cancer in otherwise resistant tumours.

Cell Death and Disease (2011) 2, e245; doi:10.1038/cddis.2011.131; published online 22 December 2011

Subject Category: Cancer

Apoptosis is a controlled form of cell death, which is essential for normal development and the maintenance of tissue homeostasis in multicellular organisms. Deregulation of apoptosis disturbs the balance between the proliferation and death of cells, with too much proliferation leading to tumour formation and cancer. ${ }^{1}$ Upregulation of anti-apoptotic cellular FLICE inhibitory proteins (c-FLIPs) have been demonstrated in different types of cancer. ${ }^{2}$ Several mRNA isoforms of C-FLIP have been reported, from which three protein isoforms are expressed, namely c-FLIP $P_{\text {long }}\left(c-F L I P_{L}\right)$, $c-$ FLIP $_{\text {short }}\left(\mathrm{c}-\mathrm{FLIP}_{\mathrm{S}}\right)$ and c-FLIP $\left.\mathrm{Paji}\left(\mathrm{c}-\mathrm{FLIP}_{\mathrm{R}}\right)\right)^{3-5} \mathrm{c}-\mathrm{FLIP}$ resembles procaspase-8 with two death effector domains (DEDs) and a catalytically inactive caspase-like domain. The two shorter isoforms, c-FLIP and c-FLIP ${ }_{\mathrm{R}}$, mainly consist of the DEDs with a truncated C-terminal end. Interestingly, a functional single nucleotide polymorphism (SNP) regulates whether C-FLIP or FLIP ${ }_{\mathrm{R}}$ is produced in humans. ${ }^{6}$ The FLIP proteins can modulate apoptosis in close proximity to death receptors such as CD95 (Fas/Apo-1), tumour necrosis factor (TNF) receptor-1 (TNF-R1), TNF-related apoptosis inducing ligand (TRAIL) receptor-1 (TRAIL-R1; DR4) and TRAIL-R2 (DR5). ${ }^{7}$ The so called death-inducing signalling complex (DISC) is formed upon triggering of death receptors and thereby activation of their intracellular domains, which allows recruitment of an adaptor protein such as FADD (Fasassociated death domain-containing protein), which, in turn, can recruit the DED-containing proteins procaspase-8, procaspase-10 and the different c-FLIP isoforms. ${ }^{7}$ The initiator caspases 8 and 10 become activated upon dimerisation and proteolytic cleavage at the DISC, initiating a signalling cascade resulting in apoptosis. ${ }^{8-10}$ The DEDs of c-FLIP allow recruitment to the DISC, ${ }^{11}$ where cleavage of initiator caspases can be prevented and caspase activation is consequently inhibited. ${ }^{3,5,12}$ Although the short isoforms c-FLIP $\mathrm{S}_{\mathrm{S}}$ and $\mathrm{C}-\mathrm{FLIP}_{\mathrm{R}}$ have been shown to solely possess anti-apoptotic functions, ${ }^{5,11,12}$ the role of $c-F L I P_{L}$ has been more controversially discussed. ${ }^{3,13} \mathrm{C}-\mathrm{FLIP}_{\mathrm{L}}$ was shown to exert either proapoptotic or anti-apoptotic functions depending on its expression level ${ }^{14,15}$ and on the strength of CD95 receptor stimulation. ${ }^{16}$ Moderate expression of $C-F L I P_{L}$ was shown to promote apoptosis only upon strong receptor stimulation or in combination with overexpression of C-FLIPS or $\mathrm{C}-\mathrm{FLIP}_{\mathrm{R}}$. In contrast, high levels of $\mathrm{C}-\mathrm{FLIP}_{\mathrm{L}}$ diminished the sensitivity for CD95-induced apoptosis and caspase-8 activation. ${ }^{16}$

As numerous reports have demonstrated upregulation of c-FLIP in various cancers, prominently Hodgkin's lymphoma $^{17,18}$ and melanoma, ${ }^{4,19}$ may allow tumours avoiding

\footnotetext{
${ }^{1}$ Laboratory of Systems-oriented Immunology and Inflammation Research, Institute of Molecular and Clinical Immunology, Otto-von-Guericke-University Magdeburg and Department of Immune Control, Helmholtz Centre for Infection Research, Inhoffenstr 7, D-38124 Braunschweig, Germany; ${ }^{2}$ Institute of Medical Microbiology and Hospital Hygiene, Heinrich-Heine-University, Universitaetsstr 1, D-40225 Duesseldorf, Germany and ${ }^{3}$ Department of Urology, Heinrich Heine University, D-40225 Duesseldorf, Germany

*Corresponding author: I Schmitz, Laboratory of Systems-oriented Immunology and Inflammation Research, Institute of Molecular and Clinical Immunology, Otto-von-Guericke-University Magdeburg and Division of Immune Control, Helmholtz Centre for Infection Research, Inhoffenstr 7, D-38124 Braunschweig, Germany. Tel: + 49531 61813500; Fax: + 49531 61813599; E-mail: ingo.schmitz@ @elmholtz-hzi.de

${ }^{4}$ Current address: German Sport University Cologne, Am Sportpark Muengersdorf 6, 50933 Cologne, Germany. Keywords: apoptosis; bladder cancer; cancer therapy resistance; death receptor

Abbreviations: c-FLIP, cellular FLICE inhibitory protein; c-FLIP,$c-F L I P_{\text {long; }}$ c-FLIP,$c$ c-FLIP Raji; c-FLIP,$c$ c-FLIP short; CHX, cycloheximide; DED, death effector domain; DISC, death-inducing signalling complex; FADD, Fas-associated death domain; NUC, normal urothelial cells; shRNA, short hairpin RNA; SNP, single nucleotide polymorphism; TNF, tumour necrosis factor; TNF-R1, tumour necrosis factor receptor 1; TRAIL, TNF-related apoptosis-inducing ligand

Received 24.10.11; accepted 16.11.11; Edited by A Stephanou
} 
endogenous mechanisms inducing apoptosis and especially escaping immune surveillance. ${ }^{2,20,21}$ Moreover, upregulation of c-FLIP has been suggested as a possible mechanism for resistance to therapeutic triggering of death receptors and the extrinsic apoptotic pathway, which is studied as a promising approach to cancer therapy. ${ }^{22}$ In particular, the death receptor ligand TRAIL selectively induces apoptosis in various tumour cells in vivo without affecting cells in normal tissues. ${ }^{23,24}$ However, more recent studies described resistance against TRAIL-induced apoptosis in many primary tumour cells. $^{25}$

CD95 and TRAIL have also been implicated in the pathogenesis and response to therapy in bladder cancer. 25,26 In 2010, cancer of the urinary bladder was the fourth most common cancer in men in the United States as well as in the European Union (EU) and more than $90 \%$ of the cases were of the urothelial carcinoma histological subtype. Bladder cancer is primarily treated by surgery. Immunotherapy by BCG is commonly used to prevent recurrences and is thought to be mediated partly by effects of neutrophil-derived TRAIL on residual tumour cells. ${ }^{27}$ Cisplatin-based chemotherapy is used for the treatment of advanced stage cases, but is only moderately efficacious. In 2008, almost 30000 patients died of bladder cancer in the EU. Because of the high morbidity and mortality of bladder cancers, there is an urgent need for improved treatment strategies and in particular, for understanding the mechanisms underlying resistance to immunotherapy and chemotherapy.

The expression and function of c-FLIP in urothelial cancer are of obvious interest in that context, but few studies are available to date. One immunohistochemical study described an association of strong c-FLIP expression with tumour progression in bladder cancer, but curiously a lack of expression in normal urothelium. ${ }^{28}$ As many cancers retained CD95 expression, the authors suggested that c-FLIP might contribute to resistance against CD95-induced apoptosis. However, no functional experiments were performed. In contrast, another study provided evidence that C-FLIP $P_{L}$ might contribute to TRAIL resistance of some urothelial carcinoma cell lines. ${ }^{29}$ Unfortunately, these studies have not been followed up to date. In particular, the contribution of the different C-FLIP isoforms to protection of CD95- and TRAILmediated apoptosis in urothelial carcinoma cells has not been studied. Therefore, we examined the expression of $\mathrm{C}-\mathrm{FLIP}_{\mathrm{L}}$ and $\mathrm{c}-\mathrm{FLIP}_{\mathrm{S}}$ in primary tumours and cell lines and their contribution to resistance against death receptor-mediated apoptosis in urothelial carcinoma cell lines in detail. Surprisingly, we observed that $C-F_{L} P_{L}$ was decreased in primary tumours and cell lines compared with normal urothelial tissue and cells. Nevertheless, urothelial carcinoma cell lines were resistant towards apoptosis-induction by CD95L or TRAIL, and required prevention of protein synthesis for sensitisation, indicating that short-lived proteins such as c-FLIP may contribute to resistance. Indeed, specific downregulation of c-FLIP by RNA interference using short hairpin RNAs (shRNAs) sensitised urothelial carcinoma cell lines towards both CD95- and TRAIL-mediated apoptosis. Thus, despite diminished expression, c-FLIP proteins appear to remain important resistance factors with respect to apoptosis-based therapies in bladder cancer.

\section{Results}

C-FLIP $\mathrm{L}_{\mathrm{L}}$ expression is decreased in urothelial carcinoma. We first analysed the expression of $C-F L I P L$ and $c-F L I P S$ mRNA in urothelial carcinoma samples. C-FLIPL mRNA levels were moderately but significantly decreased in tumour samples compared with normal urothelial tissue (Figure 1a). Similarly, the expression of $c-F_{L} P_{L}$ as quantified by real-time PCR was lower in urothelial carcinoma cell lines than in cultured normal urothelial cells

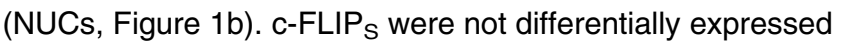
between either tissues or cell lines (Figures 1a and b). Of note, a few tissue samples did not express c-FLIPS at all, most likely due to the presence of a functional SNP (rs10190751 A/G) in the $c$-FLIP gene, which determines whether C-FLIP $_{\mathrm{R}}$ or FLIP is produced. $^{6}$ In the context of the former study, ${ }^{6}$ however, we had not observed significant changes in the distribution of this SNP between bladder cancer patients and controls (data not shown).

To find a suitable cell model for determining whether even lower levels of c-FLIP can mediate resistance towards death receptor-mediated apoptosis and to investigate the role of c-FLIP splice variants in urothelial carcinoma, we next analysed the urothelial carcinoma cell lines $639 \mathrm{v}, \mathrm{SD}, 647 \mathrm{v}$, VMCub1, BFTC905 and J82 for c-FLIP protein expression. Both protein isoforms, C-FLIP $P_{L}$ and $c-F_{S}$, were readily observed in these cell lines except for $639 \mathrm{v}$ cells, in which cFLIP $_{S}$ was hardly detectable (Figure 1c). Furthermore, the cell lines were analysed by flow cytometry analysis and western blotting to evaluate death receptor expression (Supplementary Figure 1), sensitivity towards CD95L-induced apoptosis (data not shown), and the expression of proteins involved in the 'extrinsic' and 'intrinsic' apoptotic pathways (Supplementary Figure 2). The cell lines VMCub1 and SD were chosen for further experiments based on their death receptor and c-FLIP expression profile.

Cycloheximide (CHX) sensitises urothelial carcinoma cell lines towards CD95L- and TRAIL-induced apoptosis. To investigate whether the extrinsic pathway can be triggered in VMCub1 and SD, the death receptor expression of these cell lines was analysed by surface staining of the death receptors CD95, TRAIL-R1 (DR4), TRAIL-R2 (DR5) and TNF-R1. VMCub1 cells had a moderate-to-high expression of CD95, TRAIL-R2 and TNF-R1, whereas SD cells had a moderate-to-high expression of all the four death receptors (Figure 1d). To assess CD95L- and TRAIL-induced apoptosis of VMCub1 and SD, cells were stimulated with the ligands for 16 and $24 \mathrm{~h}$, respectively, before measurement of DNA fragmentation. Both urothelial carcinoma cell lines showed low sensitivity towards CD95L-induced (Figure 2a) as well as TRAIL-induced apoptosis (Figure 2b). However, VMCub1 and SD cells were strongly sensitised towards apoptosis by addition of the protein translation inhibitor $\mathrm{CHX}$ (Figures 2a and $b$ ). An additional measure of apoptosis is caspase processing as the initiator caspase-8 and the effector caspase- 3 are activated by proteolytic cleavage. For this purpose, VMCub1 and SD cells were stimulated with CD95L for up to $8 \mathrm{~h}$ (Figure $2 \mathrm{c}$ ) or TRAIL for up to $24 \mathrm{~h}$ (Figure $2 \mathrm{~d}$ ) in 

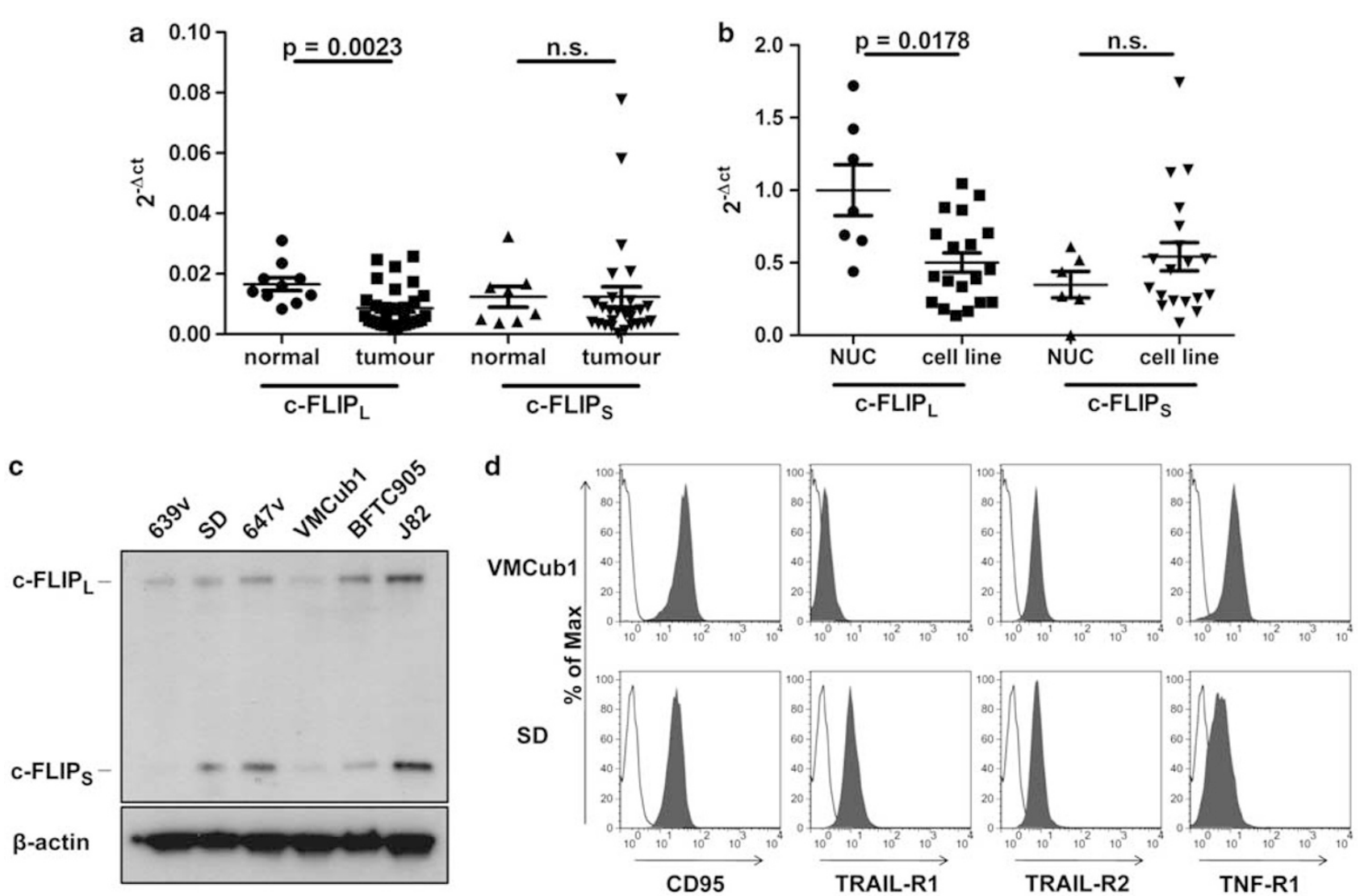

Figure 1 (a) Quantification of c-FLIP and c-FLIPS mRNA levels in normal urothelial tissues (normal; $n=10$ ) and urothelial carcinoma samples (tumour; $n=28$ ) by real-time RT-PCR. Values were normalised to GAPDH expression. Horizontal lines represent the mean; error bars display the standard deviation. Statistical analyses were performed by two-tailed Mann-Whitney U-tests. (b) Quantification of c-FLIP and c-FLIPS mRNA levels in NUCs $(n=7)$ and urothelial cell lines (cell line; $n=19$ ) by real-time PCR. Values were normalised to TBP expression levels. Horizontal lines represent the mean; error bars display the standard deviation. Statistical analyses were performed by two-tailed Mann-Whitney U-tests. (c) Western blot analysis of c-FLIP protein expression in the indicated urothelial cell lines. $\beta$-Actin was used as the loading control. (d) VMCub1 and SD cell surface expression of the death receptors CD95, TRAlL-R1, TRAIL-R2 and TNF-R1 analysed by flow cytometry. Unstained cells are shown in white

the absence or presence of $\mathrm{CHX}$, followed by western blot analysis to examine caspase cleavage as an early event during apoptosis. There was only minimal cleavage of caspase- 8 and caspase-3 after stimulation with CD95L or TRAIL alone, whereas the presence of $\mathrm{CHX}$ caused substantial cleavage of both caspases. Taken together, our results indicate that short-lived proteins have an important role in protecting the urothelial carcinoma cells against apoptosis. In particular, at least one short-lived protein protects against apoptosis at a step before caspase- 8 activation, for which c-FLIP is the most likely candidate. ${ }^{30,31}$

c-FLIP overexpression protects urothelial carcinoma cells against apoptosis. To ensure that c-FLIP is indeed a short-lived protein in urothelial carcinoma cells, we followed protein levels of c-FLIP after treatment of VMCub1 and SD cells with $\mathrm{CHX}$ (Figure 3a). Consistent with previous reports, ${ }^{30,31}$ a rapid turnover of both $\mathrm{c}-$ FLIP $_{\mathrm{L}}$ and $\mathrm{c}-\mathrm{FLIP}_{\mathrm{S}}$ was observed, with $c-F_{L} P_{L}$ being slightly more stable. This finding suggests that the c-FLIP proteins are likely candidates for the short-lived proteins responsible for the reduced sensitivity against apoptosis in the urothelial carcinoma cell lines. As it is known that all three c-FLIP splice variants can inhibit death receptor-mediated apoptosis, ${ }^{3,5,32}$ we investigated whether overexpression of any C-FLIP isoform could protect VMCub1 and SD cells against apoptosis. The three C-FLIP splice variants $C-$ FLIP $_{\mathrm{L}}$, c-FLIP $S_{S}$ and $c-$ FLIP $_{R}$ were cloned into a vector, which allows tracking of transfected cells by IRES-driven GFP expression. The generated constructs were first transiently transfected into 293T cells to verify that the respective c-FLIP variants and in addition GFP were expressed at the protein level (Figure 3b). We then transiently transfected VMCub1 and SD cells with either the empty vector or one of the c-FLIP constructs, stimulated the cells with CD95L and examined the intracellular levels of active caspase-3 by flow cytometry. Indeed, both VMCub1 (Figures $3 \mathrm{c}$ and $\mathrm{d}$ ) and SD cells (Figures $3 e$ and $f$ ) were protected by overexpression of any c-FLIP variant against CD95L-mediated apoptosis. The protective effect of c-FLIP overexpression was also confirmed by staining of transfected and stimulated cells using tetramethylrhodamine ethyl ester (TMRE; data not shown).

Knockdown of c-FLIP sensitises urothelial carcinoma cells for CD95L-induced apoptosis. As high expression of 


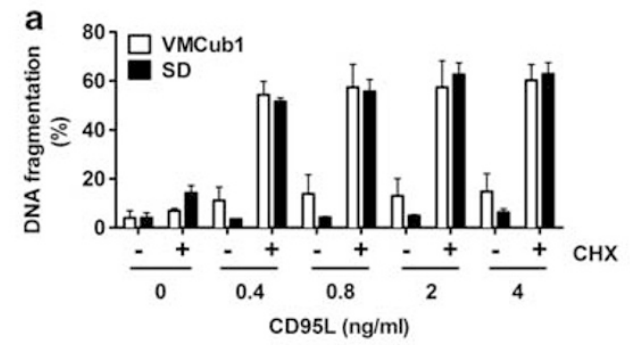

b

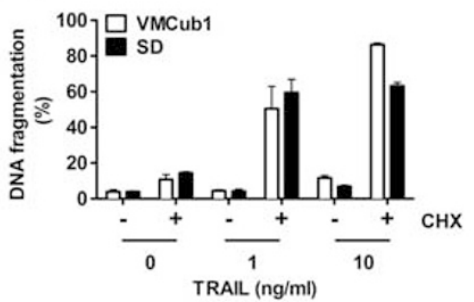

VMCub1

d

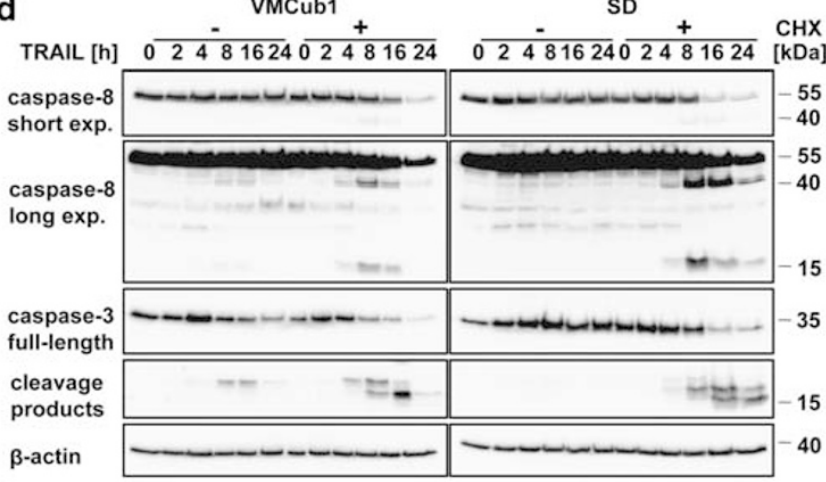

Figure 2 Urothelial carcinoma cell lines VMCub1 and SD are sensitised to apoptosis by CHX. (a and $\mathbf{b})$ For analysis of apoptosis sensitivity, urothelial carcinoma cells were left untreated or stimulated for $16 \mathrm{~h}$ with the indicated concentrations of CD95L (a) or TRAIL (b) in the presence or absence of $10 \mu \mathrm{g} / \mathrm{ml} \mathrm{CHX.} \mathrm{The} \mathrm{amount} \mathrm{of} \mathrm{apoptotic}$ cells was determined by quantification of sub-G1 DNA content by flow cytometry. Measurements are displayed as the mean of at least three independent experiments \pm S.D. (c and d) VMCub1 and SD cells were left untreated or stimulated for the times as indicated with $0.4 \mathrm{ng} / \mathrm{ml} \mathrm{CD95L}$ (c) or $25 \mathrm{ng} / \mathrm{ml} \mathrm{TRAlL} \mathrm{(d)} \mathrm{in} \mathrm{presence} \mathrm{or} \mathrm{absence} \mathrm{of} 10 \mu \mathrm{g} / \mathrm{ml}$ $\mathrm{CHX}$. Cleavage of caspase- 8 and caspase- 3 was analysed by western blot with $\beta$-actin as the loading control

all c-FLIP isoforms is reported to block death receptormediated apoptosis, $3,5,32$ we followed a loss-of-function approach to determine specific functions of the different c-FLIP splice variants. We used RNA interference and stably transduced the VMCub1 and SD cells with lentiviral shRNA constructs targeting $\mathrm{C}-\mathrm{FLIP}_{\mathrm{L}}, \mathrm{c}-\mathrm{FLIP}_{\mathrm{S}}$ or both c-FLIP $\mathrm{L}_{\mathrm{S}}$ isoforms (Supplementary Figure 3). The knockdown of C-FLIP $\mathrm{L}_{\mathrm{L}}$ was less efficient than the $\mathrm{c}-$ FLIP $_{\mathrm{S}}$ and $\mathrm{c}-\mathrm{FLIP}_{\mathrm{L} / \mathrm{S}}$ knockdowns. Still, we observed more efficient shRNA knockdowns in the urothelial cell lines VMCub1 and SD than in the human T-cell line CEM. ${ }^{33}$

As assessed by measurement of DNA fragmentation, stimulation of VMCub1 and SD knockdown cells with increasing concentrations of CD95L led to elevated levels of apoptosis in the c-FLIP L/S $_{\text {d }}$ double knockdown cells (Figures $4 a$ and b). The effect was most prominent for the SD cells expressing shRNAs targeting both $C-F_{L} P_{L}$ and $C-F L I P S$, where the extent of apoptosis was comparable to those of wild-type SD cells treated with $\mathrm{CHX}$ (Figure 2a). This finding strongly supports the argument that $\mathrm{C}$-FLIP is a prominent short-lived anti-apoptotic protein in these cells. Moreover, SD cells with knockdown of C-FLIP $\mathrm{L}_{\mathrm{L}}$ alone were highly susceptible towards CD95L-induced apoptosis, whereas SD cells with knockdown of c-FLIP alone remained resistant (Figure $4 b$ ). VMCub1 cells with single knockdown of $c-$ FLIP $_{L}$ or $c-F L I P S$ showed a mild, but statistically significant increase in CD95Lmediated apoptosis (Figure 4a). The effects of $c-F L I P L$ or

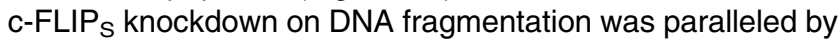
effects on caspase processing. The most extensive cleavage of caspase-8 and caspase-3 was observed in VMCub1 and
SD c-FLIP L/S $_{\text {double knockdown cells as well as SD c-FLIP }}$ single knockdown cells, where the proform of caspase-8 was extensively processed to the cleavage products p41/43 and p18 of caspase-8 as well as p17/20 of caspase-3 (Figures 4c and d). In contrast, both caspase- 8 and caspase- 3 remained uncleaved in VMCub1 and SD cells transduced with scramble shRNA. Thus, c-FLIP proteins protect the urothelial carcinoma cells against CD95L-induced apoptosis. Furthermore, the long c-FLIP isoform appears to be more important.

Urothelial carcinoma cells are sensitised for TRAILinduced apoptosis by knockdown of c-FLIP. We also investigated the effect of isoform-specific c-FLIP knockdown on TRAIL-induced apoptosis in VMCub1 and SD cells. Susceptibility to TRAIL-induced apoptosis was assayed by measurement of DNA fragmentation. Comparable to the CD95L-induced apoptosis assays, the VMCub1 and SD c-FLIP $\mathrm{L}_{\mathrm{S}}$ double knockdown cells as well as the SD C-FLIP single knockdown cells showed elevated levels of apoptosis upon TRAIL stimulation (Figures $5 a$ and $b$ ). To assess activation of caspase- 8 and caspase-3, cells were stimulated with TRAIL for up to $24 \mathrm{~h}$. The cleavage products of caspase- 8 and caspase- 3 were readily detected in the VMCub1 c-FLIPL/S double knockdown cells upon TRAIL stimulation, indicating activation of both caspases (Figure 5c). Small amounts of both processed caspases could also be detected in VMCub1 cells expressing shRNAs targeting C-FLIP $\mathrm{L}_{\mathrm{L}}$ or $\mathrm{C}_{-}$FLIPS $_{\mathrm{S}}$ alone, suggesting that the c-FLIP isoforms are not complementary. In SD cells, the cleavage products $\mathrm{p} 18 /$ $41 / 43$ of caspase- 8 and p17/20 of caspase-3 were clearly 
a

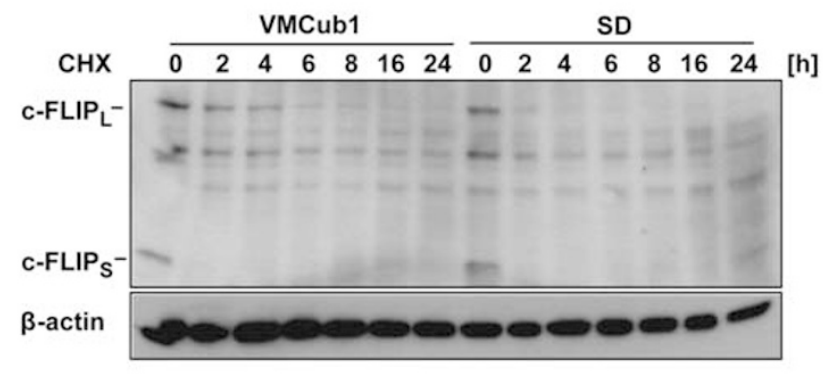

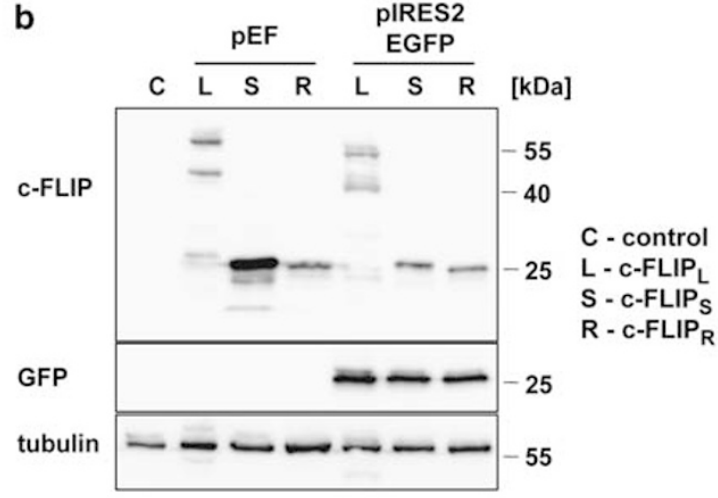
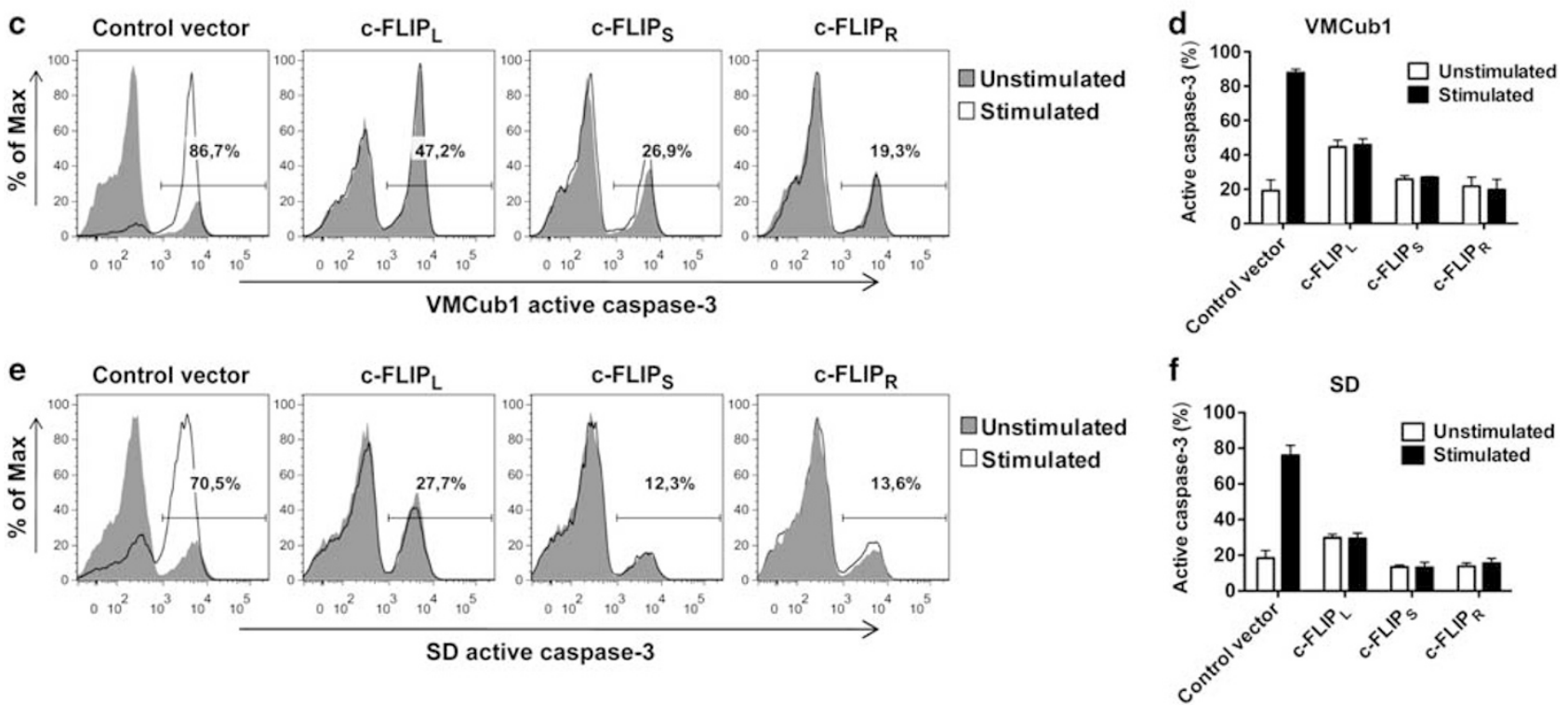

Figure 3 Overexpression of c-FLIP isoforms protects urothelial carcinoma cells against apoptosis. (a) VMCub1 and SD cells were treated with $10 \mu \mathrm{g} / \mathrm{ml} \mathrm{CHX}$ for up to $24 \mathrm{~h}$. c-FLIP protein expression was determined by western blotting. $\beta$-Actin served as a loading control. (b) Original pEF-vectors and the generated pIRES2EGFP-c-FLIP constructs were transiently transfected into $293 \mathrm{~T}$ cells. The c-FLIP and GFP protein expression was analysed by western blotting with tubulin as the loading control. (c-f) pIRES2EGFP-c-FLIP constructs were transiently overexpressed in VMCub1 cells (c and $\mathbf{d}$ ) or SD cells (e and f). The urothelial carcinoma cells were left untreated or stimulated with $1 \mathrm{ng} / \mathrm{ml} \mathrm{CD95L}$ for $4 \mathrm{~h}$. Sensitivity to apoptosis in transfected cells was analysed by flow cytometry. Intracellular staining of active caspase-3 was used as a marker for apoptotic cells and the transfected cells were identified by GFP expression. (c and $\mathbf{e}$ ) Histograms are representative for three independent experiments with percentages shown for stimulated samples. ( $\mathbf{d}$ and $\mathbf{f}$ ) Data are shown as the percentages within the GFP-positive population with the mean of three independent experiments \pm S.D.

detected in C-FLIP $\mathrm{L}_{\mathrm{S}}$ double knockdown cells and in C-FLIP single knockdown cells. Moreover, the proform of both caspase-8 and caspase-3 was extensively degraded in SD cells expressing shRNAs targeting both $\mathrm{c}-\mathrm{FLIP} \mathrm{P}_{\mathrm{L}}$ and $\mathrm{C}-\mathrm{FLIP} \mathrm{S}$ at later time points (Figure $5 \mathrm{~d}$ ). In contrast, both caspases remained uncleaved in VMCub1 and SD cells stably transduced with scramble shRNAs, suggesting that c-FLIP proteins confer broad resistance to death receptor activation in urothelial carcinoma.

\section{Discussion}

Resistance to cell death is one hallmark of cancer cells. ${ }^{1}$ Accordingly, proapoptotic factors such as Bax are often downregulated in tumours, whereas anti-apoptotic molecules such as $\mathrm{Bcl}-2, \mathrm{Bcl}-\mathrm{x}_{\mathrm{L}}$ or IAPs tend to be upregulated. Similarly, high c-FLIP expression has been observed in various cancers, for example, in Hodgkin's lymphoma ${ }^{17,18}$ and melanoma. ${ }^{4}$ It is therefore intriguing that we observed a lower expression of the C-FLIP $\mathrm{L}_{\mathrm{L}}$ isoform in urothelial tumour tissues compared with normal tissues, which was recapitulated in frequency and extent in urothelial carcinoma cell lines compared with NUCs. To our knowledge, there is only one previous study of c-FLIP expression in bladder cancer tissues, which was performed by immunohistochemistry. ${ }^{28}$ These authors observed c-FLIP expression in $81 \%$ of 53 studied urothelial cancers, predominantly in cases with higher T-stage and worse clinical course. It is bit curious that c-FLIP could not be detected in $20 \%$ of the cancers and normal urothelium by immunohistochemistry in this study, as in our hands c-FLIP protein and its according mRNA was detectable in all carcinoma cell lines and in NUCs. Interestingly, in the study 

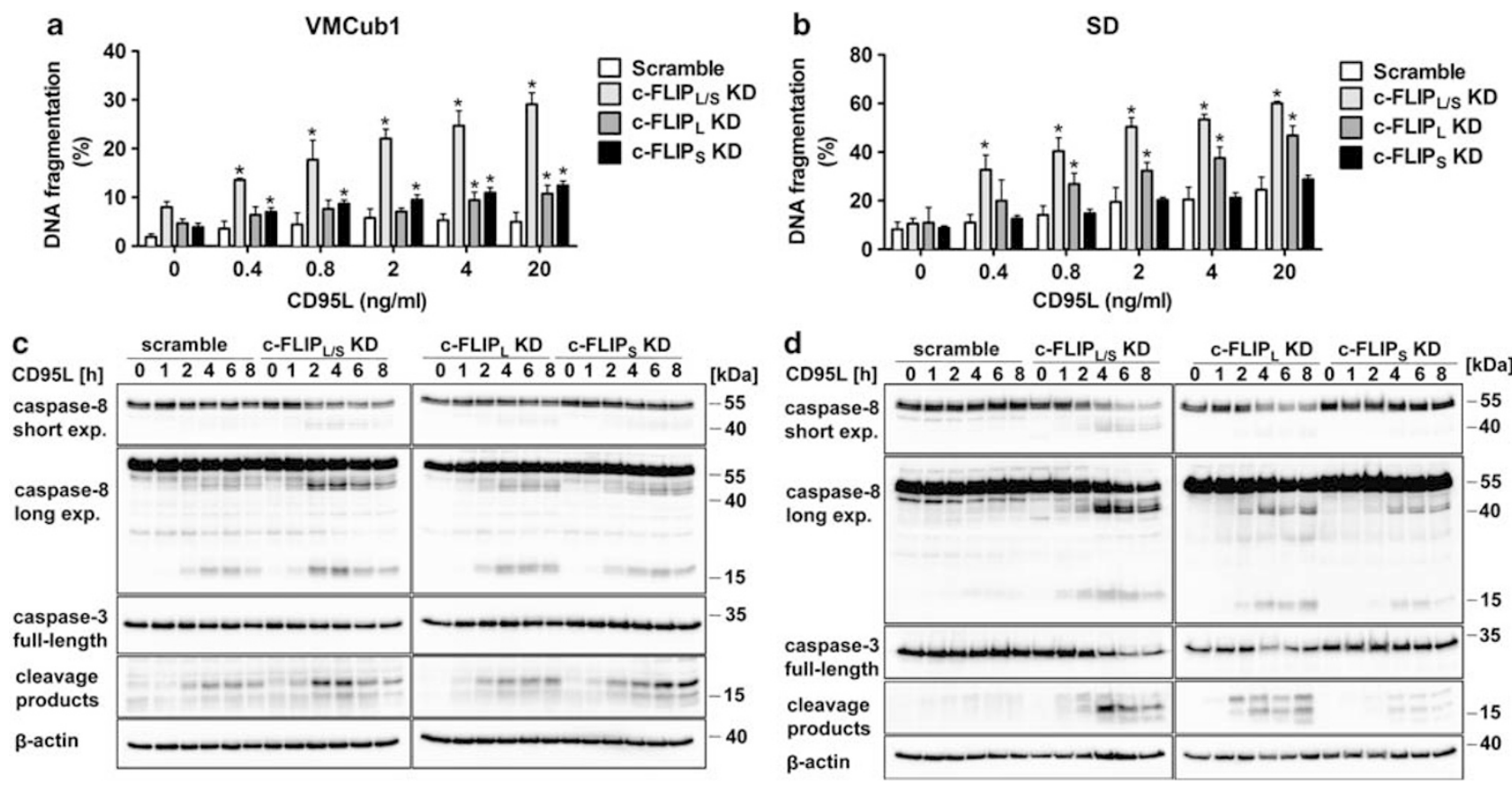

Figure 4 Knockdown of C-FLIP and c-FLIPS sensitises urothelial carcinoma cells to CD95L-induced apoptosis. (a and $\mathbf{b}$ ) For analysis of apoptosis sensitivity, VMCub1 (a) or SD cells (b) stably transduced with the indicated shRNAs against c-FLIPL, C-FLIPS or both isoforms (c-FLIP $P_{L S}$ ) were left untreated or stimulated for $16 \mathrm{~h}$ with the indicated concentrations of CD95L. The amount of apoptotic cells was quantified by DNA fragmentation analysed by flow cytometry. Data are shown as the mean of four measurements \pm S.D. Statistical analyses was performed by two-tailed Mann-Whitney U-tests, The symbol ${ }^{*}$ indicates $P<0.05$ with respect to scramble controls. (c and $\mathbf{d}$ )

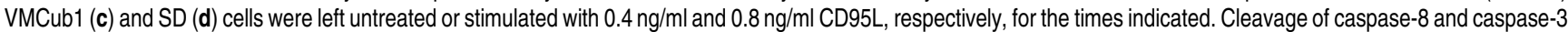
was analysed by western blot. Expression of $\beta$-actin is presented as the loading control
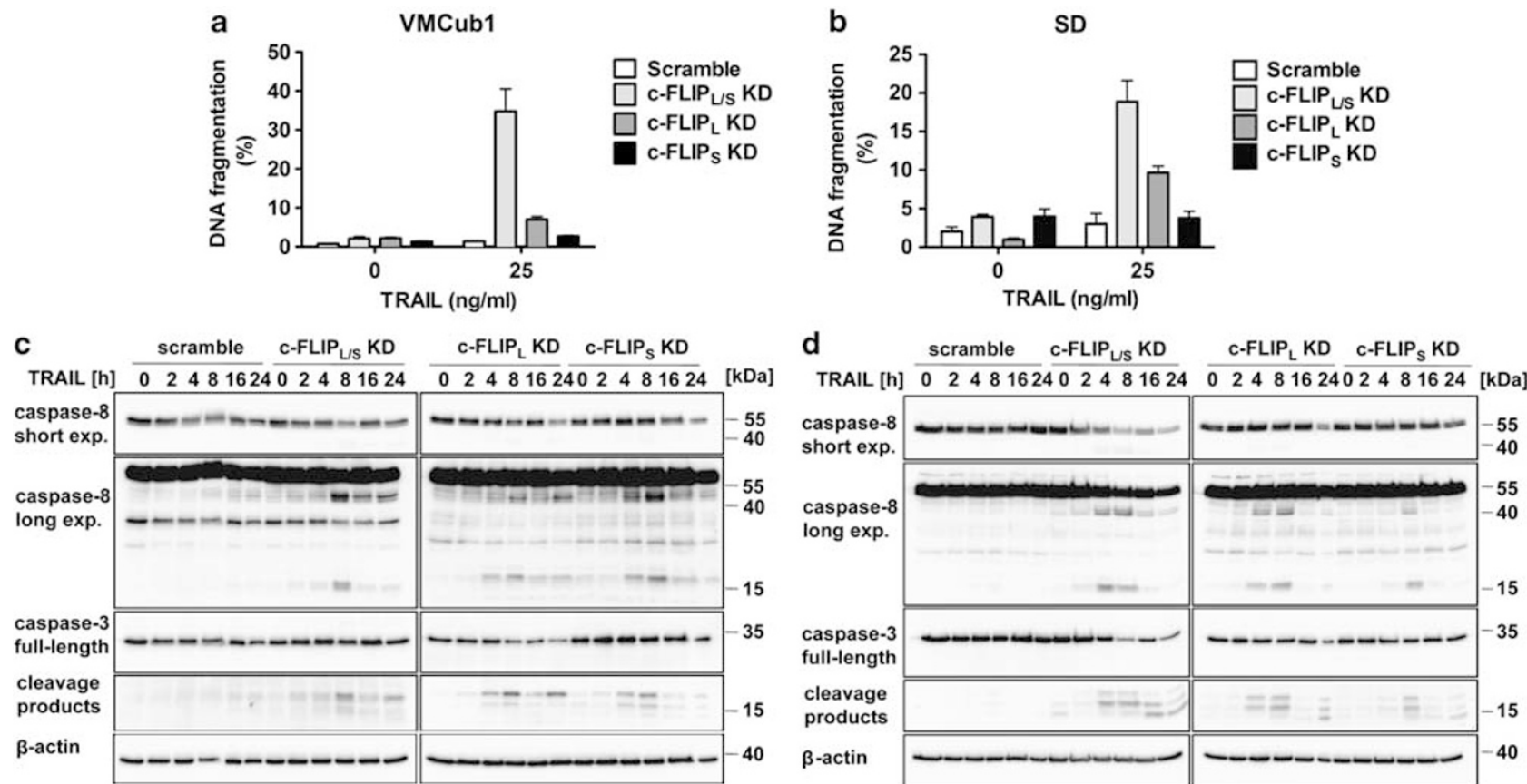

Figure 5 Effect of c-FLIP isoform knockdown on the susceptibility of urothelial carcinoma cells to TRAIL-induced apoptosis. (a and b) VMCub1 (a) or SD cells (b) with knockdown of C-FLIP,$c-F L I P_{S}$ or both isoforms (c-FLIP ${ }_{L S}$ ) were left untreated or stimulated with $25 \mathrm{ng} / \mathrm{ml}$ TRAIL for $24 \mathrm{~h}$. The amount of apoptotic cells was quantified by measuring the sub-G1 DNA content by flow cytometry. Data are shown as the mean of at least three measurements \pm S.D. (c and d) For analysis of caspase-8 and caspase-3 cleavage, VMCub1 (c) and SD cells (d) were left untreated or stimulated with $25 \mathrm{ng} / \mathrm{ml}$ TRAIL for the times as indicated. Lysates were analysed by western blot with $\beta$-actin as the loading control 
by Korkolopoulou et al., ${ }^{28}$ concurrent expression of CD95, CD95L and c-FLIP was observed in 22 cases, suggesting a function of $\mathrm{c}$-FLIP in protecting the tumours against CD95mediated apoptosis. Our present study provides direct evidence for this function. Specific downregulation of c-FLIP by RNA interference using shRNAs sensitised urothelial carcinoma cell lines towards both CD95L- and TRAILmediated apoptosis. Consistently, a function of c-FLIP in resistance to TRAIL has been observed by others. ${ }^{29}$

Although independent studies on c-FLIP mRNA and protein expression in tissue samples may be helpful, it will likely remain difficult to distinguish the different c-FLIP isoforms in tissue samples by immunohistochemistry. Our finding that they are differentially expressed in urothelial carcinoma accentuates the question as to their individual function. The two splice variants C-FLIP $\mathrm{L}_{\mathrm{L}}$ and $\mathrm{c}-\mathrm{FLIP}_{\mathrm{S}}$ inhibit death receptormediated apoptosis by different mechanisms ${ }^{12}$ and can even have opposing functions on cell death. ${ }^{15,16}$ In contrast to lymphoid cells, in which c-FLIPs has a dominant role in protection against CD95-mediated apoptosis, ${ }^{33}$ our data suggest that the short isoform has a more auxiliary function in urothelial carcinoma. In the SD model system, isoformspecific knockdown of $C-F L I P_{L}$ was sufficient to sensitise cells for apoptosis induction by death ligands. Importantly, knockdown of both isoforms in SD cells resulted in similar apoptosis levels as co-treatment with $\mathrm{CHX}$, suggesting that c-FLIP is the major short-lived anti-apoptotic protein in these cells. In VMCub1 cells, however, single knockdowns did not sensitise for apoptosis and complete inhibition of c-FLIP expression resulted in partial sensitisation compared with $\mathrm{CHX}$ co-treatment, indicating that additional factors contribute to apoptosis resistance in this cell line. Of note, we detected elevated expression of $\mathrm{Bcl}-2$ and $\mathrm{Bcl}-\mathrm{x}_{\mathrm{L}}$ in VMCub1 cells, which may contribute to resistance against death receptormediated apoptosis.

Our observation that $\mathrm{C}-\mathrm{FLIP}_{\mathrm{L}}$ is downregulated in urothelial carcinoma appears counterintuitive at first as one would expect that high expression of an anti-apoptotic molecule is generally beneficial for tumour cell survival. However, apart from apoptosis induction, signalling through CD95 exerts additional functions, especially in resistant cells. Thus, CD95 in some instances promotes tumour growth as well as motility and invasiveness of tumour cells. ${ }^{34,35}$ These functions are mediated by activation of the NF- $\kappa \mathrm{B}$ and JNK pathways, which have been shown to be inhibited by high c-FLIP expression. ${ }^{34,36,37}$ Therefore, cancer cells may have to fine-tune c-FLIP expression to balance apoptosis resistance on the one hand with non-apoptotic death receptor signalling via NF- $\kappa \mathrm{B}$ and other pathways on the other hand. Moreover, $C-F L I P_{L}$ has been shown to have a dual role in apoptosis signalling being anti-apoptotic at high expression levels and proapoptotic at low expression levels. ${ }^{16}$ Even more complicated, C-FLIPL acquires proapoptotic function in the presence of high c-FLIPS expression. Thus, as they also express $c-F_{L} P_{S}$, urothelial carcinoma cells may benefit from downregulation of $C-F L I P_{L}$ by preventing its proapoptotic activity. Finally, an optimal C-FLIP $P_{L}$ and $c-$ FLIP $_{S}$ expression level and ratio may be required to prevent activation of the Ripoptosome, a recently described cell death-initiating multiprotein complex. ${ }^{38,39}$ Depending on its molecular composition, the Ripoptosome initiates either apoptosis or necroptosis. Activation of the kinases RIP1 and RIP3 leads to necroptosis, whereas their inactivation by caspase-8-mediated cleavage favours apoptosis. Importantly, c-FLIPS inhibits caspase-8 activity within the Ripoptosome and may, therefore, promote necroptosis. ${ }^{38}$ In contrast, caspase- 8 and C-FLIP $\mathrm{L}_{\mathrm{L}}$ inhibit necroptosis, a cell death mode, which leads to inflammation. As the recruited inflammatory cells may result in tumour cell elimination, blocking Ripoptosome function by $c-F_{L} P_{L}$ may be important for urothelial carcinoma development.

Taken together, we have shown that c-FLIP, and in particular the c-FLIP $P_{L}$ splice variant, is an important apoptosis resistance factor in urothelial carcinomas. Therefore, targeting C-FLIP in CD95L- or TRAIL-based therapies may be a promising approach to treat urothelial tumours. Moreover, c-FLIP may constitute a factor in the development of resistance against immunotherapy and chemotherapy used in the treatment of bladder cancer, in so far as their efficacy depends on activation of death receptors.

\section{Materials and Methods}

Patients and tissue samples. Tissue samples were obtained from patients undergoing cystectomy for urothelial carcinoma at the Department of Urology of the Heinrich-Heine-University Duesseldorf between 1995 and 2004. Twenty-four patients were male and seven were female; average age at surgery was 69.9 years (range 54-94 years). The samples were selected to comprise all stages and included three tumours each staged as pTa or pT1, 5 staged as pT2, 11 staged as pT3 and 7 staged as pT4. Lymph node metastases were present in seven patients. The lymph node status was not determined further for two additional patients with $>$ pT2 cancers. Eleven cancers were graded as G2 and 20 as G3. Morphologically normal tissue from 10 patients was used for comparison. The use of patient tissues was approved by the Ethics Committee of the Medical Faculty of the Heinrich-Heine-University Duesseldorf.

Cell culture and transient transfections. Urothelial carcinoma cell lines 639v, SD, 647v, VMCub1, BFTC905 and J82 as well as human embryonic kidney cells (293T) were cultured in Dulbecco's modified Eagle's medium (DMEM high glucose; Invitrogen, Carlsbad, CA, USA) supplemented with $10 \%$ fetal calf serum (PAA Laboratories, Coelbe, Germany) and $50 \mu \mathrm{g} / \mathrm{ml}$ of each penicillin and streptomycin (Invitrogen). Transient transfections of 293T, VMCub1 and SD cells were performed with JetPEI (Polyplus transfection, Illkirch, France) or Lipofectamine 2000 reagent (Invitrogen) according to the manufacturer's instructions. Normal urothelial cells were isolated and cultured according to the method of Southgate et al. with slight modifications as described. ${ }^{40}$

Lentiviral infection of cells. c-FLIP MISSION TRC shRNA Target Set cloned into the lentiviral vector pLKO.1 was purchased from Sigma (Sigma-Aldrich Chemie, Steinheim, Germany). Generation of c-FLIP short shRNA was previously described. ${ }^{33}$ Lentiviral vectors were co-transfected with the envelope vector pMD2.G (Addgene no. 12259) and the gag-pol expression plasmid pCMV_dR8.2dvpr (Addgene no. 8455) into 293T cells as described in 'Cell culture and transient transfections'. Lentiviruses (LVs) were collected 36 and $60 \mathrm{~h}$ after transfection. Crude virus was filtered through $0.45 \mu \mathrm{m}$ PVDF filters (Millipore, Billerica, MA, USA), concentrated by ultracentrifugation at $100000 \times g$ for 60 min at $4^{\circ} \mathrm{C}$ and stored at $-80^{\circ} \mathrm{C}$ until further use. Titres were determined by infection of HeLa cells with serial dilutions of LV stocks and $5 \mu \mathrm{g} / \mathrm{ml}$ polybrene (Sigma-Aldrich Chemie). Specific knockdown of the various c-FLIP isoforms was verified by western blot analysis. SD and VMCub1 cells were infected by adding LVs and $5 \mu \mathrm{g} /$ $\mathrm{ml}$ polybrene to $2 \times 10^{6}$ cells. Cells were centrifuged for $1 \mathrm{~h}$ at $860 \mathrm{~g}$ and then incubated over night. Stably transfected clones were selected by limited dilution in medium containing $4 \mu \mathrm{g} / \mathrm{ml}$ puromycin (Sigma-Aldrich Chemie).

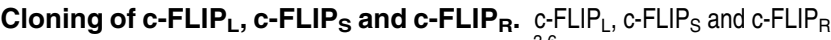
were cloned using previously described plasmids ${ }^{3,6}$ as PCR templates. PCR reactions were performed with Phusion flash proofreading polymerase (Finnzymes, 
Vantaa, Finland) using the following primers: c-FLIP forward: $5^{\prime}$-GGCTAGCCATG TCTGCTGAAGTCATCCA-3'; c-FLIP reverse $5^{\prime}$-GCCCGGGCTTATGTGTAGGAG AGGATAA-3'; c-FLIPS reverse 5'-GCCCGGGCTCACATGGAACAATTTCCAA-3'; c-FLIP $P_{R}$ reverse $5^{\prime}$-GCCCGGGCTCATGCTGGGATTCCATATG- $3^{\prime}$. Thereafter cloning into the expression vector pIRES2EGFP (Clontech, Mountain View, CA, USA) was performed by using restriction digestion with Smal and Nhel (New England Biolabs, Ipswich, MA, USA).

Western blot analysis. For western blot analysis, cells were lysed in TPNE buffer (PBS adjusted to $300 \mathrm{mM} \mathrm{NaCl}, 1 \%$ Triton-X100, 2 mM EDTA, $1 \mathrm{mM}$ PMSF and $1 \mu \mathrm{g} / \mathrm{ml}$ each of leupeptin, aprotinin, chymostatin and pepstatin A). Twenty micrograms of postnuclear supernatant protein as determined by the BCA method (Pierce Biotechnology, Rockford, IL, USA) were separated by $12 \%$ SDS-PAGE, blotted onto a PVDF membrane (Amersham, Freiburg, Germany) and blocked with $5 \%$ non-fat dry milk in TBS/Tween (0.05\% Tween-20 in TBS). The blots were washed with TBS/Tween and were thereafter incubated with specific antibodies overnight at $4{ }^{\circ} \mathrm{C}$. The following day, blots were washed with TBS/Tween, incubated with horseradish peroxidase-coupled secondary antibodies $(1: 20000)$ for $1 \mathrm{~h}$ at room temperature, washed again and developed with a chemiluminescence reagent (Pierce Biotechnology). For stripping, blots were incubated in Re-Blot mild solution (Millipore) according to the manufacturer's protocols.

The antibodies used for western blotting were $\beta$-actin (AC-74; Sigma-Aldrich Chemie), caspase-3 (CPP32; R\&D Systems, Minneapolis, MN, USA), cleaved caspase-3 (Asp175; Cell Signalling Technology, Danvers, MA, USA), caspase-8 (12F5; kind gift of Dr. K Schulze-Osthoff, Tuebingen, Germany), caspase-7 (SigmaAldrich Chemie), c-FLIP (NF6; Enzo Life Sciences, Loerrach, Germany), Bcl-xL (clone 44) and XIAP (clone 48; BD Biosciences, Heidelberg, Germany). Furthermore, anti-FADD (1F7), anti-Bax (6A7) and anti-Bak were purchased from Upstate (Hamburg, Germany). Anti-Bcl-2 (C-2) and HRPO-conjugated goat antirabbit IgG were from Santa Cruz Biotechnology (Santa Cruz, CA, USA). HRPOconjugated goat anti-rat $\lg G$, donkey anti-goat $\lg G$, goat anti-mouse $\lg G 1$, IgG2a and IgG2b were from Southern Biotechnology Associates (Birmingham, AL, USA)

Quantitative real-time PCR. Total RNA was isolated from 5-10 $\times 10^{6}$ cells with the RNeasy kit (Qiagen, Hilden, Germany). Real-time PCR was carried out on an Applied Biosystems 7300 Real-Time PCR system using the QuantiTect SYBR Green RT-PCR Kit (Qiagen) according to the manufacturer's instructions. Measurements were run in triplicates and normalised to GAPDH or TBP values. Amplification primers for real-time PCR are as follows:

c-FLIP $P_{L}$ forward $5^{\prime}$-CCTAGGAATCTGCCTGATAATCGA-3';

c-FLIP $P_{L}$ reverse $5^{\prime}$-TGGGATATACCATGCATACTGAGATG-3';

c-FLIP forward 5'-GCAGCAATCCAAAAGAGTCTCA-3';

c-FLIP reverse $^{\prime}$-ATTTCCAAGAATTTTCAGATCAGGA-3';

GAPDH forward 5'-ATCACCATCTTCCAGGAGCGAGATC-3';

GAPDH reverse 5'-GGCAGAGATGATGACCCTTTTGGC-3';

TBP forward $5^{\prime}$-CGAAACGCCGAATATAATCC-3';

TBP reverse $5^{\prime}$-CGTGGCTCTCTTATCCTCA-3'.

Flow cytometry assays. Cell surface staining and cytotoxicity assays were performed as described previously. ${ }^{30}$ For assaying apoptosis, $2.5 \times 10^{5}$ cells per well were left untreated or were stimulated in 6-well-plates for $16 \mathrm{~h}$ with CD95L or for $24 \mathrm{~h}$ with TRAIL at the indicated concentrations. The following antibodies were used for cell surface staining: TNF-R1 (kind gift of Dr. H Wajant, Wuerzburg, Germany), TRAIL-R1 (Enzo Life Sciences) and TRAIL-R2 (PE-conjugated; eBioscience, San Diego, CA, USA). CD95 surface staining was performed with 2R2 antibody (kind gift of Dr. K Schulze-Osthoff). TNF-R1, TRAIL-R1 and CD95 antibodies were labelled with PE-conjugated goat-anti-mouse secondary antibody (Jackson ImmunoResearch, Suffolk, UK). For assaying the effect of c-FLIP overexpression on apoptosis sensitivity, VMCub1 and SD cells were transiently transfected $\left(2.5 \times 10^{5}\right.$ cells in 6-well-plates) with empty pIRES2EGFP vector (Clontech) or the $c-F L I P_{L}, C-F L I P_{S}, C-F L I P_{R}$ constructs (see above). Cells were left untreated or were stimulated with $1 \mathrm{ng} / \mathrm{ml} \mathrm{CD} 95 \mathrm{~L}$ for $4 \mathrm{~h}$, followed by intracellular staining of active caspase-3 (BD Biosciences) or TMRE staining (Enzo Life Sciences) according to the manufacturer's manual.

Statistical analysis. All statistical analyses were performed by nonparametric Mann-Whitney U-tests to determine statistical significance using GraphPad Prism (Graph-Pad-Software Inc., La Jolla, CA, USA). Standard deviation and standard error of the mean were used as error bars.

\section{Conflict of Interest}

The authors declare no conflict of interest.

Acknowledgements. We thank Stephanie Grosch, Dominique Gollasch and Sabrina Schumann for expert technical assistance. We are grateful to Dr. Klaus Schulze-Osthoff and Dr. Harald Wajant for supplying antibodies and to Dr. Klaus Pfeffer for his support. This work was supported by the German Research Foundation (DFG, SCHM1586/2-1) and Deutsche Krebshilfe (108962).

1. Igney $\mathrm{FH}, \mathrm{Krammer} \mathrm{PH}$. Death and anti-death: tumour resistance to apoptosis. Nat Rev Cancer 2002; 2: 277-288.

2. Shirley S, Micheau O. Targeting c-FLIP in cancer. Cancer Lett 2010; e-pub ahead of print 9 November 2010; doi:10.1016/j.canlet.2010.10.009.

3. Scaffidi C, Schmitz I, Krammer PH, Peter ME. The role of C-FLIP in modulation of CD95-induced apoptosis. J Biol Chem 1999; 274: 1541-1548.

4. Irmler M, Thome M, Hahne M, Schneider P, Hofmann K, Steiner V et al. Inhibition of death receptor signals by cellular FLIP. Nature 1997; 388: 190-195.

5. Golks A, Brenner D, Fritsch C, Krammer PH, Lavrik IN. C-FLIPR, a new regulator of death receptor-induced apoptosis. J Biol Chem 2005; 280: 14507-14513.

6. Ueffing N, Singh KK, Christians A, Thorns C, Feller AC, Nagl F et al. A single nucleotide polymorphism determines protein isoform production of the human c-FLIP protein. Blood 2009; 114: 572-579.

7. Peter ME, Krammer PH. The CD95(APO-1/Fas) DISC and beyond. Cell Death Differ 2003; 10: 26-35.

8. Boatright KM, Renatus M, Scott FL, Sperandio S, Shin H, Pedersen IM et al. A unified model for apical caspase activation. Mol Cell 2003; 11: 529-541.

9. Chang DW, Xing Z, Capacio VL, Peter ME, Yang X. Interdimer processing mechanism of procaspase-8 activation. EMBO J 2003; 22: 4132-4142.

10. Donepudi M, Mac Sweeney A, Briand C, Grutter MG. Insights into the regulatory mechanism for caspase-8 activation. Mol Cell 2003; 11: 543-549.

11. Ueffing N, Keil E, Freund C, Kuhne R, Schulze-Osthoff K, Schmitz I. Mutational analyses of c-FLIPR, the only murine short FLIP isoform, reveal requirements for DISC recruitment. Cell Death Differ 2008; 15: 773-782.

12. Krueger A, Schmitz I, Baumann S, Krammer PH, Kirchhoff S. Cellular FLICE-inhibitory protein splice variants inhibit different steps of caspase-8 activation at the CD95 deathinducing signalling complex. J Biol Chem 2001; 276: 20633-20640.

13. Budd RC, Yeh WC, Tschopp J. cFLIP regulation of lymphocyte activation and development. Nat Rev Immunol 2006; 6: 196-204.

14. Chang DW, Xing Z, Pan Y, Algeciras-Schimnich A, Barnhart BC, Yaish-Ohad S et al. $c$ - $F L I P(L)$ is a dual function regulator for caspase-8 activation and CD95-mediated apoptosis. EMBO J 2002; 21: 3704-3714

15. Micheau O, Thome M, Schneider P, Holler N, Tschopp J, Nicholson DW et al. The long form of FLIP is an activator of caspase-8 at the Fas death-inducing signaling complex. J Biol Chem 2002; 277: 45162-45171.

16. Fricker N, Beaudouin J, Richter P, Eils R, Krammer PH, Lavrik IN. Model-based dissection of CD95 signaling dynamics reveals both a pro- and antiapoptotic role of c-FLIPL. J Cell Biol 2010; 190: 377-389.

17. Dutton A, O'Neil JD, Milner AE, Reynolds GM, Starczynski J, Crocker J et al. Expression of the cellular FLICE-inhibitory protein (c-FLIP) protects Hodgkin's lymphoma cells from autonomous Fas-mediated death. Proc Natl Acad Sci USA 2004; 101: 6611-6616.

18. Mathas S, Lietz A, Anagnostopoulos I, Hummel F, Wiesner B, Janz M et al. c-FLIP mediates resistance of Hodgkin/Reed-Sternberg cells to death receptor-induced apoptosis. $J$ Exp Med 2004; 199: 1041-1052.

19. Griffith TS, Chin WA, Jackson GC, Lynch DH, Kubin MZ. Intracellular regulation of TRAILinduced apoptosis in human melanoma cells. J Immunol 1998; 161: 2833-2840.

20. Medema JP, de Jong J, van Hall T, Melief CJ, Offringa R. Immune escape of tumors in vivo by expression of cellular FLICE-inhibitory protein. J Exp Med 1999; 190: 1033-1038.

21. Djerbi M, Screpanti V, Catrina Al, Bogen B, Biberfeld P, Grandien A. The inhibitor of death receptor signaling, FLICE-inhibitory protein defines a new class of tumor progression factors. J Exp Med 1999; 190: 1025-1032.

22. Wajant $\mathrm{H}$, Gerspach J, Pfizenmaier K. Engineering death receptor ligands for cancer therapy. Cancer Lett 2011; e-pub ahead of print 13 January 2011; doi:10.1016/..canlet.2010.12.019.

23. Walczak H, Miller RE, Ariail K, Gliniak B, Griffith TS, Kubin M et al. Tumoricidal activity of tumor necrosis factor-related apoptosis-inducing ligand in vivo. Nat Med 1999; 5: 157-163.

24. Wiley SR, Schooley K, Smolak PJ, Din WS, Huang CP, Nicholl JK et al. Identification and characterization of a new member of the TNF family that induces apoptosis. Immunity 1995; 3: 673-682

25. Voelkel-Johnson C. TRAIL-mediated signaling in prostate, bladder and renal cancer. Nat Rev Urol 2011; 8: 417-427.

26. O'Kane HF, Watson CJ, Johnston SR, Petak I, Watson RW, Williamson KE. Targeting death receptors in bladder, prostate and renal cancer. J Urol 2006; 175: 432-438.

27. Rosevear HM, Lightfoot AJ, O'Donnell MA, Griffith TS. The role of neutrophils and TNF-related apoptosis-inducing ligand (TRAIL) in bacillus Calmette-Guerin (BCG) immunotherapy for urothelial carcinoma of the bladder. Cancer Metastasis Rev 2009; 28: 345-353. 
28. Korkolopoulou P, Goudopoulou A, Voutsinas G, Thomas-Tsagli E, Kapralos P, Patsouris $E$ et al. C-FLIP expression in bladder urothelial carcinomas: its role in resistance to Fasmediated apoptosis and clinicopathologic correlations. Urology 2004; 63: 1198-1204.

29. Steele LP, Georgopoulos NT, Southgate J, Selby PJ, Trejdosiewicz LK. Differential susceptibility to TRAIL of normal versus malignant human urothelial cells. Cell Death Differ 2006; 13: 1564-1576.

30. Schmitz I, Weyd H, Krueger A, Baumann S, Fas SC, Krammer PH et al. Resistance of shor term activated $T$ cells to CD95-mediated apoptosis correlates with de novo protein synthesis of c-FLIPshort. J Immunol 2004; 172: 2194-2200.

31. Fulda S, Meyer E, Debatin KM. Metabolic inhibitors sensitise for CD95 (APO-1/Fas) induced apoptosis by down-regulating Fas-associated death domain-like interleukin 1-converting enzyme inhibitory protein expression. Cancer Res 2000; 60: 3947-3956.

32. Krueger A, Baumann S, Krammer PH, Kirchhoff S. FLICE-inhibitory proteins: regulators of death receptor-mediated apoptosis. Mol Cell Biol 2001; 21: 8247-8254.

33. Ueffing N, Schuster M, Keil E, Schulze-Osthoff K, Schmitz I. Up-regulation of c-FLIP short by NFAT contributes to apoptosis resistance of short-term activated T cells. Blood 2008 112: $690-698$.

34. Barnhart BC, Legembre P, Pietras E, Bubici C, Franzoso G, Peter ME. CD95 ligand induces motility and invasiveness of apoptosis-resistant tumor cells. EMBO J 2004; 23 : 3175-3185.

35. Chen L, Park SM, Tumanov AV, Hau A, Sawada K, Feig C et al. CD95 promotes tumour growth. Nature 2010; 465: 492-496.
36. Legembre P, Barnhart BC, Peter ME. The relevance of NF-kappaB for CD95 signaling in tumor cells. Cell Cycle 2004; 3: 1235-1239.

37. Kreuz S, Siegmund D, Rumpf JJ, Samel D, Leverkus M, Janssen $O$ et al. NFkappaB activation by Fas is mediated through FADD, caspase-8, and RIP and is inhibited by FLIP. J Cell Biol 2004; 166: 369-380.

38. Feoktistova M, Geserick P, Kellert B, Dimitrova DP, Langlais $C$, Hupe M et al. clAPs block Ripoptosome formation, a RIP1/caspase-8 containing intracellular cell death complex differentially regulated by cFLIP isoforms. Mol Cell 2011; 43: 449-463.

39. Tenev $\mathrm{T}$, Bianchi $\mathrm{K}$, Darding $\mathrm{M}$, Broemer $\mathrm{M}$, Langlais $\mathrm{C}$, Wallberg $\mathrm{F}$ et al. The Ripoptosome, a signaling platform that assembles in response to genotoxic stress and loss of IAPs. Mol Cell 2011; 43: 432-448.

40. Swiatkowski S, Seifert HH, Steinhoff C, Prior A, Thievessen I, Schliess F et al. Activities of MAP-kinase pathways in normal uroepithelial cells and urothelial carcinoma cell lines. Exp Cell Res 2003; 282: 48-57.<smiles>[13CH][131In]</smiles>

Cell Death and Disease is an open-access journal published by Nature Publishing Group. This work is licensed under the Creative Commons Attribution-Noncommercial-Share Alike 3.0 Unported License. To view a copy of this license, visit http:// creativecommons.org/licenses/by-nc-sa/3.0/

Supplementary Information accompanies the paper on Cell Death and Disease website (http://www.nature.com/cddis) 